\title{
ARTÍCULOS
}

Sometido 10.03.2016. Aprobado 19.10.2016

Evaluado por el sistema double blind review. Editor Científico: José Henrique de Faria

DOI:http://dx.doi.org/10.1590/So034-759020170502

\section{EL ROL DEL CONSEJO DE ADMINISTRACIÓN EN LA ÉTICA EMPRESARIAL EN PAÍSES DE LATINOAMÉRICA}

\author{
Papel do conselho de administração na ética empresarial em países da \\ América Latina
}

The role of the board of directors in corporate ethics in Latin American countries

\begin{abstract}
RESUMEN
El presente trabajo analiza los valores éticos fomentados en los códigos de ética en las empresas latinoamericanas, e identifica la influencia de la composición del Consejo de Administración (CA). En particular, la presencia de diversidad de género, consejeros independientes y extranjeros en el CA. El estudio, realizado sobre las 100 empresas cotizadas más grandes de Latinoamérica, muestra que las empresas estudiadas elaboran códigos de conducta con valores éticos que mejoran las relaciones con sus stakeholders. No obstante, prevalece el establecimiento de valores que protegen a la organización del empleado y la imposición de sanciones. Por otro lado, la regresión efectuada indica que la presencia de mujeres y consejeros independientes en el CA afecta de manera significativa y positiva al contenido de los códigos de ética, mientras que la presencia de consejeros extranjeros incide negativamente. Los hallazgos de esta investigación permiten avanzar en la escasa literatura existente sobre la ética empresarial en el contexto latinoamericano.
\end{abstract}

PALABRAS-CLAVE | Código de ética, diversidad de género, consejeros independientes, consejeros extranjeros, responsabilidad social.

ARTURO HARO-DE-ROSARIO

arturo.haro@ual.es

Profesor de la Universidad de Almería, Departamento de Economía y Empresa - La Cañada-Almería, España

\section{MARÍA DEL MAR GÁLVEZ-}

\section{RODRÍGUEZ}

margalvez@ual.es

Profesora de la Universidad de Almería, Departamento de Economía y Empresa - La Cañada -Almería, España

\section{ALEJANDRO SÁEZ-MARTÍN}

alejandro.saez@ual.es

Profesor de la Universidad de Almería, Departamento de Economía y Empresa - La Cañada-Almería, España

\section{CARMEN CABA-PÉREZ}

ccaba@ual.es

Profesora de la Universidad

de Almería, Departamento

de Economía y Empresa - La

Cañada-Almería, España

\section{RESUMO}

O presente trabalho analisa os valores éticos fomentados nos códigos de ética das empresas latino-americanas e identifica a influência da composição do Conselho de Administração (CA) - em particular, a presença de diversidade de gênero e de conselheiros independentes e estrangeiros no CA. $O$ estudo, realizado com as 100 maiores empresas latino-americanas cotadas em bolsa, mostra que as empresas estudadas elaboram códigos de conduta com valores éticos que melhoram as relações com seus stakeholders. No entanto, prevalece o estabelecimento de valores que protegem a organização do funcionário, além da imposição de sanções. Por outro lado, a regressão efetuada indica que a presença de mulheres e conselheiros independentes no CA afeta de maneira significativa e positiva o conteúdo dos códigos de ética, enquanto a presença de conselheiros estrangeiros tem um impacto negativo. As descobertas desta pesquisa permitem avançar na escassa literatura existente sobre a ética empresarial no contexto latino-americano.

PALAVRAS-CHAVE / Código de ética, diversidade de gênero, conselheiros independentes, conselheiros estrangeiros, responsabilidade social.

\begin{abstract}
In the present paper, we analyze the ethical values promoted in the codes of ethics of Latin American organizations, and the influence of the composition of the Board of Directors (BD) in this regard is identified. In particular, the presence of gender diversity, independent directors and foreign directors in the $B D$. The study was conducted on the 100 largest listed companies in Latin America. The results indicate that the companies studied elaborate codes of conduct with ethical values that enhance the relationships with their stakeholders. However, prevailing the establishment of values that protect the organization from the employee, and the imposition of sanctions. On the other hand, the regression analysis indicates a positive and significant influence of women and independent directors of the BD in the content of codes of ethics however, the effect of foreign directors in this respect is negative. The research findings allow advance the sparse literature on business ethics in the Latin American context.
\end{abstract}

KEYWORDS / Code of ethics, gender diversity, independent directors, foreign directors, social responsibility. 


\section{INTRODUCCIÓN}

La conducta ética de las empresas en los países de Latinoamérica ha cobrado gran relevancia a consecuencia de los diversos casos de fraude, entre otros, por parte de empleados y gerencia (Arruda, 1997, 2009). En este sentido, se entiende que una empresa mantiene una conducta ética cuando actúa bajo un conjunto de valores éticos y principios básicos (Weiss, 1991). Asimismo, la creciente internacionalización de las empresas latinoamericanas ha provocado que tanto sus inversores como sus clientes extranjeros exijan la implementación de mecanismos de buenas prácticas (Bedicks \& Arruda, 2005; Calderón, 2011).

Entre los mecanismos de buenas prácticas más utilizados se encuentra el código de ética (Adelstein \& Clegg, 2016). En términos generales, consiste en un documento escrito y formal que contiene valores cuya finalidad es garantizar la conducta ética de la organización hacia sus diferentes grupos de interés o stakeholders y, comunicar cuál es la conducta que la organización espera de sus empleados (Kaptein \& Wempe, 2002). Para Wotruba, Chonko y Loe (2001) el código de ética presenta tres objetivos principales, demostrar interés por la ética, transmitir los valores a todos los miembros de la organización y, tener un impacto en la conducta ética de los mismos.

No obstante, la mera existencia de un código de ética no garantiza su eficacia (Wotruba et al., 2001). Para Erwin (2010) ésta dependerá de la coherencia entre la conducta ética de la organización y la demanda de buenas prácticas de los stakeholders. Es por ello que autores como Gilley, Robertson y Mazur (2010) señalan que su elaboración debe realizarse de manera sistemática, comprehensiva, inclusiva y estratégica. En cuanto a la finalidad estratégica, Kaptein y Schwartz (2008) ponen de manifiesto la importancia de la adecuada integración de valores que ayuden no solo a la adecuada conducta de los empleados sino también a la mejora en los resultados de gestión de la organización.

Con respecto a los responsables de la elaboración y fomento de los códigos de ética, cabe señalar el papel de los miembros del consejo de administración (CA, en adelante) ya que son los responsables de la toma de decisiones clave en la organización, como es la política de buenas prácticas contra la corrupción en la organización (Coonjohn \& Lodin, 2011; GarcíaSánchez, Rodríguez-Domínguez, \& Frías-Aceituno, 2015). A este respecto, con el fin de evitar comportamientos corruptos, tales como sobornos a funcionarios públicos, tráfico de influencias, fraude, chantaje, soborno electoral y juego ilegal (Office of Transport Safety Investigations - OTSI, 2010), el CA debe estar compuesto por miembros que fomenten el comportamiento ético en la cultura de la organización, sobre todo en países con un elevado nivel de corrupción, como es el caso de los países latinoamericanos (Arruda, 1997; Calderón, 2011).

Centrándonos en la composición del CA, Elias (2003) y Barrientos (2005) señalan la necesidad de incorporar a la mujer en los procesos de elaboración e implementación de los códigos de ética, ya que muestran un mayor interés en la promoción de aspectos relacionados con la responsabilidad social de la empresa. Asimismo, la visión externa y objetiva de los consejeros independientes es también importante en la mejora de las buenas prácticas de la organización, tales como la mayor transparencia informativa (Méndez \& Gómez, 2013; RodríguezAriza, Frías-Aceituno, \& García-Rubio, 2014). Por otro lado, dada la creciente internacionalización de las empresas, cada vez es mayor el reconocimiento del papel de los consejeros extranjeros en el control de la gestión ética de la organización de los stakeholders nacionales e internacionales (Luo, 2005; Oxelheim, Gregorič, Randøy, \& Thomsen, 2013).

Aunque es extensa la literatura que ha analizado la importancia de los códigos éticos para el fomento de una adecuada conducta de los miembros de una empresa, la mayoría de los trabajos están centrados en países desarrollados como Canadá, Australia, Alemania, Suiza y Reino Unido (Bondy, Matten, \& Moon, 2004; Singh, Carasco, Svensson, Wood, \& Callaghan, 2005). Es por ello que, dado que los factores culturales e institucionales afectan a la elaboración de los códigos, Arruda (2009) destaca la necesidad de mayor atención a otros países como los latinoamericanos.

Dada la importancia de la ética en las empresas latinoamericanas y el rol del CA en la elaboración y comunicación de los códigos de ética, este trabajo presenta un doble objetivo. En primer lugar, analizar los valores éticos fomentados en los códigos de ética en las empresas latinoamericanas. En segundo lugar, identificar la influencia de la composición del CA a este respecto. Por ello, se plantean las siguientes preguntas de investigación (RQ):

RQ1: ¿Cuáles son los valores éticos más fomentados por los códigos de ética de las empresas latinoamericanas?

RQ2: ¿La presencia de diversidad de género, consejeros independientes y extranjeros en el CA influye significativamente en el contenido de los códigos de ética?

RQ2.1: En caso afirmativo, ¿Cuáles son los valores éticos que muestran diferencias significativas, atendiendo al CA?

Este trabajo contribuye tanto al ámbito académico como profesional. Desde el ámbito académico, avanza en la escasez 
de literatura concerniente al conocimiento de las conductas más valoradas para garantizar la conducta ética de la organización, así como en la influencia de la composición del CA a este respecto. Además de determinar la influencia del CA en la ética de la organización, este trabajo ofrece nuevas aportaciones en relación a la identificación de las conductas que son más o menos favorecidas en función de ciertas características de la composición del CA.

En relación al ámbito profesional, este estudio podría ser una guía para los gerentes de las empresas latinoamericanas en cuanto a la adecuada elaboración de los códigos de ética. Por otro lado, los resultados podrían ser un indicativo sobre qué tipos de consejeros dentro del CA fomentan un comportamiento ético en la organización y, a partir de ahí, poder establecer una política de selección de los miembros del CA que impulsen las buenas prácticas.

\section{LA RELEVANCIA DE LOS CÓDIGOS DE ÉTICA Y SU CONTENIDO}

Los escándalos detectados en empresas tales como Enron en el 2000, Lehman Brothers en el 2004, Barclays en el 2013 y HSBC Switzerland en el 2015, unidos a la crisis global financiera, han provocado un creciente interés por conocer los mecanismos de buenas prácticas implementados por las organizaciones y, en especial, los códigos de ética (Adelstein \& Clegg, 2016).

Para Gilley et al. (2010), el código de ética consigue el aumento de compromiso de los trabajadores en la organización, lo que revierte en su motivación y, en consecuencia, en su mayor productividad. Asimismo, destacan que su adecuado desarrollo y comunicación a los diferentes stakeholders de la organización (externos e internos) influye positivamente en la cultura y reputación de la organización. Por su parte, Lückerath-Rovers y Bos (2011) indican su contribución a la profesionalización de los miembros de la organización.

La calidad de los códigos de ética puede jugar un papel crucial en su efectividad y en su habilidad de transformar la cultura de la organización (Erwin, 2010). Es por ello que a la hora de garantizar su adecuada elaboración, Schwartz, Dunfee y Kline (2005) ponen de manifiesto que el código debe abordar los valores esenciales de la organización, donde se expresa de forma explícita qué es deseable desde un punto de vista ético. Asimismo, los autores señalan la importancia de indicar las responsabilidades y restricciones que deben asumir los miembros de la organización. Por su parte, Kaptein (2004) realizó un estudio sobre las 200 compañías más grandes del mundo con el fin de conocer cuáles son las categorías más comunes y más recomendables a la hora de desarrollar un código de ética. Con base en este trabajo identificamos cuatro categorías principales: los “principios corporativos" de la organización, los principios de "responsabilidad hacia los grupos de interés", los principios de "conducta interna” y las "sanciones e incentivos".

Los "principios corporativos" incluyen los valores generales por los cuales deben regirse la empresa y sus empleados y, por lo tanto, son la base de las relaciones con sus stakeholders. La relación ética con sus stakeholders queda expresada con mayor grado de especificad en los principios de "responsabilidad hacia los grupos de interés", donde la empresa detalla el correcto comportamiento de cara a sus clientes, inversores, sociedad, medioambiente y proveedores. Los principios de "conducta interna" están enfocados a clarificar qué es lo que se espera de los empleados. En concreto, en lo que respecta a su conducta hacia la empresa y entre ellos. Finalmente, Kaptein (2004) señala la importancia de implementar mecanismos que garanticen el cumplimento de los valores establecidos por la empresa a través de "Sanciones e incentivos".

Con base en las categorías mencionadas podemos identificar líneas de investigación concernientes al efecto de los códigos de ética en la empresa así como las centradas en su contenido. En cuanto a su efecto, cabe señalar el estudio realizado por Kaptein y Schwartz (2008), los cuales realizaron una revisión sobre 79 trabajos a este respecto que demuestra que, a pesar de que existen estudios que indican su influencia neutra, solo en un caso se detectó su efecto negativo, en la mayoría se observó una influencia significativa y positiva en la mejora de las prácticas de la empresa.

En los trabajos que analizan los contenidos de los códigos de ética se observa que, en términos generales, inicialmente existía una preocupación por integrar principios o valores en la empresa que evitasen conductas ilegales por parte de los empleados de la misma (Stevens, 1996). Sin embargo, este interés ha ido evolucionado hacia el fomento de valores concernientes con el compromiso social de la organización en relación al impacto social, económico y medioambiental de las actividades que realiza (O’Dwyer \& Madden, 2006). En el contexto latinoamericano, hay muy pocos trabajos que aborden el contenido de los códigos de ética con la excepción del estudio de Gorbaneff, Uribe y Hoyos (2012). Estos autores analizaron los códigos de siete empresas petroleras de Colombia concluyendo que los documentos aún presentan un carácter de refuerzo disciplinario y un tono legal muy diseñados para controlar la conducta de los empleados. 


\section{RELACIÓN ENTRE LA COMPOSICIÓN DEL CA Y LOS CÓDIGOS DE ÉTICA}

En líneas generales, no existe un marco regulador común sobre la composición del CA. Sin embargo, se identifican iniciativas voluntarias en países europeos como Alemania, Reino Unido, Francia o España, que incluyen recomendaciones sobre la estructura que deben mantener los CA, generalmente para el caso de empresas cotizadas (Soltani \& Maupetit, 2015). Así, sugieren que el tamaño del CA debería ser de entre 5 y 15 miembros. En cuanto a la composición del CA, los consejeros independientes representarán, con carácter general, la mitad del consejo. En casos excepcionales, se puede recomendar una proporción de, al menos, un tercio, y en el caso de CA pequeños, un mínimo de 2 consejeros independientes. Respecto a la diversidad de género, solo la iniciativa española menciona que para el año 2020 el número de consejeras debería de representar el 30\% del CA (Comisión Nacional del Mercado de Valores [CNMV], 2015). En Latinoamérica, el tamaño del CA alcanza un promedio de 10 consejeros en las empresas cotizadas, el porcentaje de consejeros independientes es del 38\% y la participación de la mujer en los CA es poco relevante (5\%) (Briano \& Saavedra, 2015).

No obstante, el nombramiento de los miembros del CA debe evitar cualquier conflicto de interés dentro del mismo, por tanto debe estar libre de cualquier tipo de sesgos, en especial de los que impliquen discriminaciones relacionadas con la diversidad de género (CNMV, 2015). Asimismo, la política de selección debe ser concreta y verificable, de manera que ningún consejero o grupo pequeño de consejeros pueda dominar la toma de decisiones del CA (Soltani \& Maupetit, 2015).

Por otro lado, los motivos por los cuales las empresas han ido adoptando códigos éticos han sido justificados en el marco de diferentes teorías. En este sentido, bajo la teoría de la legitimidad, la creación y puesta en marcha de códigos éticos por las empresas contribuyen a crear y mantener una buena reputación, generando una mayor confianza de los diferentes stakeholders (Long \& Driscoll, 2008; Rodríguez-Domínguez, Gallego-Álvarez, \& García-Sánchez, 2009). Además, Valentine y Fleischman (2008) enfatizan que el uso de los códigos éticos mejora la atención de la organización con la responsabilidad social.

Con base en la teoría de los stakeholders, las empresas no solo deben responder a la demanda de buenas prácticas de los accionistas en particular, sino también al resto de stakeholders (Berrone, Surroca, \& Tribó, 2007). Es por ello que el código de ética es considerado como un instrumento organizacional donde se expresa la voluntad de la empresa por satisfacer los intereses de todas las partes implicadas (González, 2007). En esta línea, cada vez es más reconocida la importancia de la participación de los stakeholders en el desarrollo e implementación de mecanismos de buenas prácticas como los códigos de ética de las empresas (Gilley et al., 2010).

En el marco de estas teorías, se han realizado trabajos que abordan la influencia de la diversidad del CA a la hora de adoptar o promover los códigos de ética en las empresas, tales como los trabajos de Rodríguez-Domínguez et al. (2009) y García-Sánchez et al. (2015). En este sentido, el CA es un órgano de control interno que garantiza que el comportamiento de la gestión es éticamente correcto (Li, Pike, \& Haniffa, 2008). Por ello, su papel es importante a la hora de aprobary elaborar los códigos de ética (Schwartz, 2002)

Por tanto, teniendo en consideración la importancia del CA en la adecuada elaboración e implementación de los códigos de ética (Barrientos, 2005; Elias, 2003) y los trabajos que han abordado la influencia de la diversidad del CA en la adopción de dichos códigos, el presente estudio aborda si la presencia de diversidad de género, consejeros independientes y extranjeros influyen en el total de contenidos de los códigos de ética en países latinoamericanos.

\section{Diversidad de género}

La mujer es uno de los actores más involucrados en las iniciativas relacionadas con la ética de la empresa (Prieto \& Bendell, 2002; Prieto, Hadjipateras, \& Turner, 2002). Esto es debido a que la participación de la mujer en el CA es considerada como fuente de ideas e innovación en la mejora de la gobernabilidad de las empresas (Prieto-Carrón, 2008), así como en la implantación de mecanismos de buenas prácticas que mejoren los resultados y oportunidad de crecimiento de la empresa (Bear, Rahman, \& Post, 2010; Zhang, Zhu, \& Ding, 2013).

En el caso de países desarrollados, autores como GarcíaSánchez et al. (2015), encontraron una relación positiva entre la presencia de mujeres en el CA y el alcance de los códigos de ética de empresas. Por ello, ante este precedente, examinamos si la presencia de la mujer en el CA influye en el contenido de los códigos de ética de las empresas latinoamericanas, por lo que se plantea la siguiente hipótesis:

H1: La diversidad de género en el CA influye positivamente en el contenido de los códigos de ética de las empresas latinoamericanas.

\section{Consejeros independientes}

Los consejeros independientes se caracterizan por su objetividad y libertad para evaluar la gestión adecuada a la organización 
(Prado-Lorenzo \& García-Sánchez, 2010). Es por ello que son considerados como un elemento clave para la supervisión y mejora de la conducta ética de los miembros del CA (FríasAceituno, Rodríguez-Ariza \& García-Sánchez, 2013).

Su influencia positiva en la promoción de las buenas prácticas de la empresa ha sido evidenciada en trabajos relacionados con la responsabilidad social corporativa (Webb, 2004). Asimismo, en el contexto de los Estados Unidos, Europa y Canadá la tenencia de consejeros independientes en el CA ha mostrado ser significativa en la toma de decisión relativa a la elaboración de un código ético así como en el mayor alcance de su contenido (Rodríguez-Domínguez et al., 2009). Bajo estos precedentes se presenta la siguiente hipótesis:

$\mathrm{H}$ 2: Los consejeros independientes en el CA influyen positivamente en el contenido de los códigos de ética de las empresas latinoamericanas.

\section{Consejeros extranjeros}

La creciente internacionalización de las empresas ha provocado una mayor presencia de consejeros extranjeros, por lo que cada vez es más reconocida su influencia en la toma de decisiones estratégicas de la organización (Oxelheim et al., 2013). En particular, Cuadrado-Ballesteros, García-Rubio y Martínez-Ferrero (2014) evidencian su efecto positivo en la toma de iniciativas voluntarias y actuales como son la realización de actividades que mejoran el impacto social y medioambiental de la organización. En esta línea, son numerosos los estudios que han encontrado una relación positiva entre la presencia de consejeros extranjeros en el CA y la mayor transparencia informativa relativa a la responsabilidad social de la empresa (Frías-Aceituno et al., 2013; Rodríguez-Ariza et al., 2014).

Teniendo en consideración la relación positiva encontrada en trabajos previos sobre la presencia de consejeros extranjeros en el CA y los mecanismos de buenas prácticas, planteamos la siguiente hipótesis:

H3: Los consejeros extranjeros en el CA influyen positivamente en el contenido de los códigos de ética de las empresas latinoamericanas.

\section{METODOLOGÍA}

\section{Análisis de los valores éticos fomentados en los códigos de ética}

Para dar respuesta a la RQ1, se realizó un análisis de contenido deductivo, ya que la información fue codificada en base a unas categorías temáticas pre-definidas derivadas de la revisión de la literatura realizada (Abrahamson, 1983). En particular, los valores éticos analizados fueron adaptados y clasificados a partir del trabajo de Kaptein (2004). En total se utilizan 30 valores éticos agrupados en cuatro categorías principales: "principios corporativos", "responsabilidad hacia los grupos de interés", "conducta interna” y "sanciones e incentivos". Además, también se incluyó la extensión del código de ética, a través del número de páginas (Gálvez-Rodríguez, Caba-Pérez, \& López-Godoy, 2015; Kaptein, 2004) (ver primera columna de la Tabla 2).

El sistema de codificación utilizado fue de carácter dicotómico, de acuerdo con Abbott y Monsen (1979) y Wood (2000). La ausencia de subcategorías en el esquema de codificación utilizado, redujo los posibles errores de interpretación de la información, proporcionando así un análisis fiable, objetivo y sistemático (Krippendorf, 1980). No obstante, previamente a la codificación, realizada manualmente por dos codificadores independientes, se llevó a cabo una reunión para establecer la estrategia de clasificación, y al final del proceso los resultados fueron revisados para resolver las diferencias y superar posibles sesgos (Graneheim \& Lundman, 2004).

\section{Análisis de la influencia de la composición del CA en el contenido de los códigos de ética}

Atendiendo a la $R Q 2$ y RQ2.1, se ha realizado un análisis de regresión. Así, como variable dependiente se ha elaborado un índice de ética (IE). Estese determina mediante el cociente entre la sumatoria de las puntuaciones de los ítems del análisis del código de ética (ie.) y el número total de ítems:

$$
I E=\frac{\sum_{i=1}^{30} i e_{i}}{30} \times 100
$$

En cuanto a las variables independientes, se han seleccionado los factores diversidad de género, consejeros independientes y extranjeros. Además, de acuerdo con GarcíaSánchez et al. (2015), con objeto de evitar conclusiones parciales se ha incluido un set de variables de control habitualmente empleadas en la literatura (Archel, 2003; Hackston \& Milne, 1996): experiencia, tamaño y endeudamiento. En el Cuadro 1 se resumen las variables utilizadas, las unidades de medida y las relaciones esperadas. 
Cuadro 1. Factores explicativos y relaciones esperadas

\begin{tabular}{l|c|c}
\hline Factor & Medida & Relación esperada \\
\hline $\begin{array}{l}\text { Diversidad de genero } \\
\text { (Mujeres) }\end{array}$ & Presencia de mujeres en el CA (1-Sí, o-No) & Positiva \\
\hline $\begin{array}{l}\text { Consejeros independientes } \\
\text { (nndependientes) }\end{array}$ & Presencia de consejeros independientes en el CA (1-Sí, o-No) & Positiva \\
\hline $\begin{array}{l}\text { Consejeros extranjeros } \\
\text { (Extranjeros) }\end{array}$ & Presencia de consejeros extranjeros en el CA (1-Sí, o-No) & Positiva \\
\hline $\begin{array}{l}\text { Experiencia empresa } \\
\text { (Experiencia) }\end{array}$ & Lúmero de años en funcionamiento & Positiva \\
\hline $\begin{array}{l}\text { Tamaño empresa } \\
\text { (Tamaño) }\end{array}$ & Logaritmo neperiano del pasivo corriente más no corriente & Positiva \\
\hline $\begin{array}{l}\text { Endeudamiento empresa } \\
\text { (Endeudamiento) }\end{array}$ & Positiva
\end{tabular}

Una vez definidas la variable dependiente y las independientes se realiza un análisis de correlaciones. A continuación, con el fin de establecer un modelo econométrico que explique el volumen de contenido disponible en los códigos de ética, y teniendo en cuenta que la variable dependiente IE se ha definido como una variable continua comprendida entre o y 100 , se procede a aplicar un modelo de regresión Tobit. La estructura del modelo propuesto es la siguiente:

$$
\begin{aligned}
\mathrm{IE}_{\mathrm{j}}= & \beta_{\mathrm{o}}+\beta_{1} \cdot \text { Mujeres }_{\mathrm{j}}+\beta_{2} \cdot \text { Extranjeros }_{\mathrm{j}}+\beta_{3} \cdot \text { Independientes }_{\mathrm{j}}+\beta_{4} \\
& \cdot \text { Experiencia }_{\mathrm{j}}+\beta_{5} \cdot \text { Tamaño }_{\mathrm{j}}+\beta_{6} \cdot \text { Endeudamiento }_{\mathrm{j}}+\mu
\end{aligned}
$$

donde:

$\beta_{\mathrm{o}}=$ término constante.

$\beta_{\mathrm{i}}=$ parámetros de posición.

$\mu=$ perturbación aleatoria.

$\mathrm{j}=$ empresa analizada.

Tras realizar el análisis de regresión, se aplica el test U de Mann-Whitney a todos los ítems del análisis del código de ética, para identificar cuáles son los valores que se ven afectados por la diversidad de género, los consejeros independientes y los extranjeros. Este test se considera el equivalente no paramétrico al t-test, y su aplicación resulta muy apropiada para el análisis de dos grupos independientes cuando la muestra es pequeña, no se pueden asumir los supuestos de normalidad y homocedasticidad, y la variable discriminante de los dos grupos es ordinal (Sheskin, 2011). Por consiguiente, es habitualmente utilizado en estudios de sobre análisis de contenido (Criado-Jiménez, Fernández-Chulián, Husillos-Carqués, \& Larrinaga-González, 2008) y divulgación de información online (Pfeil, Arjan, \& Zaphiris, 2009; Roblyer, McDaniel, Webb, Herman, \& Witty, 2010)

\section{Muestra}

Se ha optado por seleccionar un muestreo intencional con el objetivo de analizar tendencias en los códigos de ética de las empresas latinoamericanas. Para este fin se tomó una muestra de las 100 empresas más grandes que pertenecen al ranking de las 500 empresas latinoamericanas más grandes según la revista América Economía en 2015. El tamaño muestral seleccionado está en línea con estudios similares centrados en códigos de ética de empresas de Estados Unidos (Sharbatoghlie, Mosleh, \& Shokatian, 2013).

Los códigos de ética, así como el resto de la información necesaria (informe anual, estados contables, etc.) para poder realizar los análisis previstos se han extraído de las páginas web oficiales de dichas empresas. Si atendemos al país en el que se ubican las empresas seleccionadas, se observa que la muestra se concentra principalmente en Brasil Ecuador y México siendo menor la existencia de códigos de ética en Argentina, Colombia, Ecuador y Venezuela.

En cuanto al sector de actividad, se observa que las empresas están muy repartidas. Como era de esperar, sectores como el del petróleo son muy relevantes en Latinoamérica. En cambio, sorprende que pocas empresas se dediquen a sectores como la minería o la construcción (ver Tabla 1). 
Tabla 1. Distribución por sector de actividad

\begin{tabular}{l|c}
\hline Sector & № empresas \\
\hline Alimentación y bebidas/licores & 14 \\
\hline Automotriz & 9 \\
\hline Comercio & 13 \\
\hline Construcción & 2 \\
\hline Energía eléctrica & 8 \\
\hline Minería & 5 \\
\hline Multisectorial & 10 \\
\hline Petróleo/gas & 13 \\
\hline Siderurgia/Metalurgia & 8 \\
\hline Telecomunicaciones & 12 \\
\hline Otros & 6 \\
\hline
\end{tabular}

\section{RESULTADOS}

\section{Resultados del análisis de los valores éticos más fomentados}

En términos generales, las empresas latinoamericanas contienen aproximadamente la mitad de los valores analizados (ver segunda columna de la Tabla 2). Centrándonos en cada una de las categorías, dentro de los principios corporativos, la “transparencia” y la “integridad/confianza” están presentes en la mayoría de los códigos de ética examinados. Estos resultados están en consonancia con el elevado énfasis mostrado por la transparencia en los mecanismos de rendición de cuentas de las ONG (Gálvez-Rodríguez et al., 2015). Asimismo, el interés por la confianza e integridad de las empresas latinoamericanas está en línea con las empresas públicas en España (Ayuso, 2013). En cuanto a los valores menos mencionados, cabe destacar el escaso llamamiento a la "rendición de cuentas" y a la importancia de la “diversidad de género".

Con respecto a la categoría responsabilidad hacia los grupos de interés, los valores más mencionados corresponden a aquellos que de manera más explícita tratan de fomentar la buena conducta con los clientes. A este respecto, señalar que más del $60 \%$ de los casos analizados indican la importancia de ofrecer servicios/productos de calidad y proveer los mismos de forma adecuada. Estos resultados apoyan los resultados previos del estudio de Kaptein (2004) quien señaló que las compañías más grandes del mundo, siendo en su mayoría empresas de Estados Unidos, Francia, Alemania y Japón, convergen en promocionar productos o servicios de calidad que aporten valor a los clientes. Por el contrario, es muy bajo el número de casos que enfatizan valores específicos para las buenas prácticas en relación a los inversores de la compañía.

En relación a la conducta interna, se presta gran atención a la reducción de conductas que incidan a la malversación de fondos y a la discriminación de los trabajadores. Sin embargo, es poco frecuente la inclusión de contenido para evitar las bajas injustificadas y la promoción del equilibrio entre la vida profesional y personal.

Finalmente, en lo concerniente a las sanciones e incentivos, más del $80 \%$ de las empresas analizadas indican sanciones a consecuencia del incumplimiento del contenido del código ético. Estos resultados parecen señalar que, además de la necesidad de fomentar valores que guíen la adecuada conducta de los miembros de la organización (Schwartz et al., 2005), las empresas latinoamericanas también consideran necesaria la inclusión de "castigos" que frenen el incumplimiento de las normas de conducta. Estos resultados no coinciden con los del sector de las ONG (Gálvez-Rodríguez et al., 2015), así como en el caso de las compañías más grandes a nivel mundial (Kaptein, 2004), quienes otorgan mayor importancia al establecimiento de mecanismos de seguimiento y control.

\section{Tabla 2. Análisis de los valores fomentados por los códigos de ética de las empresas latinoamericanas: La influencia de la composición del CA}

\begin{tabular}{|c|c|c|c|c|c|}
\hline & & & \multicolumn{3}{|c|}{ Z TEST } \\
\hline & Principios corporativos & $51 \%$ & $1.670^{\star}$ & - & - \\
\hline & Responsabilidad & $77 \%$ & - & - & - \\
\hline & Transparencia & $92 \%$ & - & - & $-2.106^{\star \star}$ \\
\hline & Integridad/Confianza & $92 \%$ & - & - & - \\
\hline
\end{tabular}


Tabla 2. Análisis de los valores fomentados por los códigos de ética de las empresas latinoamericanas: la influencia de la composición del CA

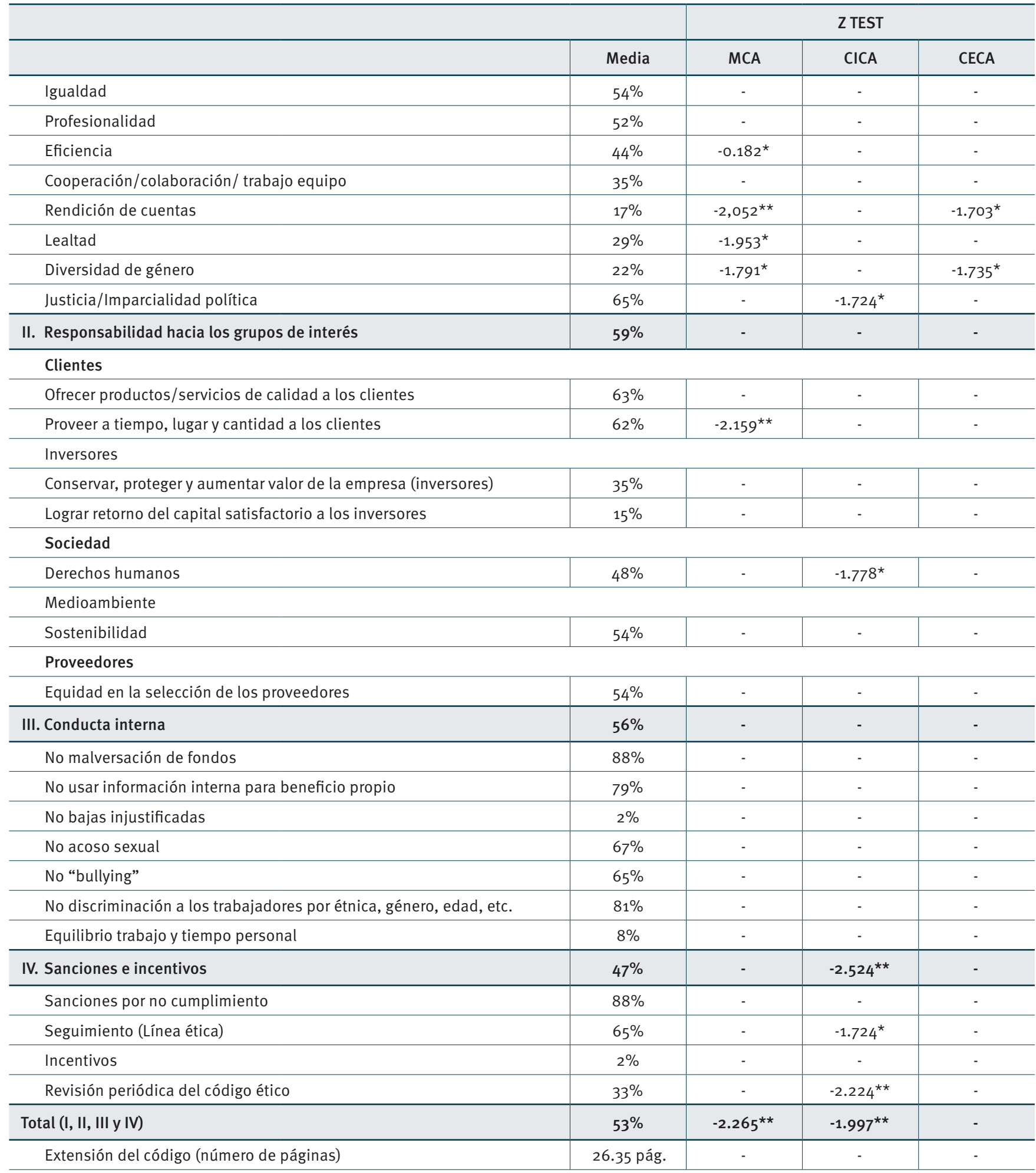

MCA: Mujeres en el Consejo de Administración; CICA: Consejeros Independientes en el Consejo de Administración; CECA: Consejeros Extranjeros en el Consejo de Administración.

* Significativo al nivel 0.1. ** Significativo al nivel 0.05. ${ }^{\star \star \star ~ S i g n i f i c a t i v o ~ a l ~ n i v e l ~} 0.01$. 


\section{Resultados del análisis de la influencia de la composición del CA}

En la Tabla 3 se muestran los resultados de la matriz de correlaciones, la cual indica que las variables mujeres y extranjeros están relacionadas significativamente con el índice de ética. En cuanto a las correlaciones entre variables independientes, solo se observan tres de grado bajo que, de acuerdo con Nachtsheim, Neter, Kutner y Wasserman (2004), no suponen ningún problema de multicolinealidad que pueda comprometer el modelo.

Tabla 3. Matriz de correlaciones de Pearson

\begin{tabular}{|c|c|c|c|c|c|c|c|}
\hline & $\mathrm{IE}$ & Mujeres & Independientes & Extranjeros & Experiencia & Tamaño & Endeudamiento \\
\hline Independientes & 0.1035 & -0.0944 & 1 & & & & \\
\hline Extranjeros & $-0.2044^{\star \star}$ & -0.0035 & 0.1169 & 1 & & & \\
\hline Tamaño & 0.0534 & -0.0083 & $-0.2625^{\star \star \star}$ & $-0.2570^{\star \star \star}$ & -0.0216 & 1 & \\
\hline Endeudamiento & -0.1040 & -0.1111 & 0.0258 & 0.0669 & -0.0466 & $0.5458^{\star \star \star}$ & 1 \\
\hline
\end{tabular}

* La correlación es significativa al nivel 0.1. ** La correlación es significativa al nivel 0.05. *** La correlación es significativa al nivel o.01.

Los resultados de la regresión Tobit confirman que el modelo propuesto resulta significativo y que tres de las seis hipótesis planteadas han resultado significativas (Tabla 4). Se observa una relación positiva y significativa entre la variable que mide la presencia de mujeres en los CA y el contenido de los códigos de ética. Por tanto, se confirma la relación esperada y se corroboran los resultados del análisis comparativo. Así, este resultado es consistente con los trabajos de Bear et al. (2010), Zhang et al. (2013) y García-Sánchez et al. (2015), que afirman que el alcance de los códigos de ética y los mecanismos de buenas prácticas son superiores en las empresas con mujeres en los CA.

En cuanto a los consejeros independientes, la regresión Tobit indica que se encuentra relacionada de forma significativa y positiva con el IE. Así, en línea con Webb (2004) y RodríguezDomínguez et al. (2009), los resultados sugieren que la presencia de consejeros independientes en el CA explica, al menos en parte, el mayor alcance de los contenidos de los códigos de ética de estas empresas.

Por otro lado, dado el efecto positivo que supone para los códigos de ética la presencia tanto de mujeres como de consejeros independientes en el CA, resulta conveniente comentar que diversos estudios que han analizado la composición de los CA (Conyon \& Mallin, 1997; Nekhili \& Gatfaoui, 2013), han encontrado una relación positiva entre la proporción de estos dos tipos de consejeros, de forma que las empresas con un mayor número de consejeros independientes incorporan más mujeres en el CA.

La variable extranjeros también ha resultado significativa. No obstante, al contrario de lo que afirman autores como Guthrie y Parker (1990) y Frías-Aceituno et al. (2013), los resultados indican que la presencia de consejeros extranjeros en el CA influye de manera negativa en el contenido de los códigos de ética. Por tanto, los CA de las empresas latinoamericanas con elevada presencia de consejeros extranjeros otorgan menos importancia a la elaboración adecuada de los contenidos de los códigos de ética.

\section{Tabla 4. Regresión Tobit}

\begin{tabular}{l|c|c|c}
\hline & \multicolumn{3}{|c}{ IE } \\
\hline LR chi2(6) & \multicolumn{3}{|c}{$14.03^{\star *}$} \\
\hline Mujeres & Coef. & Error est. & $t$ \\
\hline Independientes & 0.1153145 & 0.0599099 & $1.92^{\star \star}$ \\
\hline Extranjeros & -0.0545077 & 0.0315713 & $-1.73^{\star}$ \\
\hline Experiencia & 0.0239784 & 0.0182212 & 1.32 \\
\hline Tamaño & 0.0152141 & 0.0130908 & 1.16 \\
\hline Endeudamiento & $-2.87 \mathrm{e}-08$ & $2.25 \mathrm{e}-088$ & -1.27 \\
\hline
\end{tabular}


Los resultados de la Tabla 2 (ver última columna), nos indican en detalle qué valores de los códigos de ética son los que presentan diferencias significativas según la composición del CA. Comenzando por diversidad de género, ésta afecta principalmente a la categoría principios corporativos. En concreto, las diferencias se centran en los valores de "eficiencia", "rendición de cuentas", "lealtad" y “diversidad de género". En menor medida, la presencia de mujeres en el CA también afecta a la categoría responsabilidad hacia los grupos de interés, en particular al valor ético que menciona la importancia del cumplimiento de pedidos en términos de tiempo, lugar y cantidad. Por tanto, a pesar de las dificultades de acceso al CA que sufren las mujeres en Latinoamérica (Cárdenas et al., 2014), los resultados obtenidos están en línea con autores como Francoeur, Labelle y Sinclair-Desgagné (2008) y Mateos de Cabo, Gimeno y Nieto (2012), que sostienen que la inclusión de la mujer añade nuevos puntos de vista a los CA.

En relación a los consejeros independientes en los CA, afectan a varias categorías. Así, se observan cambios en el en valor "justicia/imparcialidad política” de la categoría principios corporativos, en el valor "derechos humanos" de la categoría responsabilidad hacia los grupos de interés, y en los valores relativos al seguimiento de sanciones y a la revisión periódica de los códigos de ética de la categoría sanciones e incentivos. Por consiguiente, estos datos van en consonancia con los de Prado-Lorenzo y García-Sánchez (2010) y los de Frías-Aceituno et al. (2013), que defienden la importancia de los consejeros independientes en la mejora de la conducta ética de las empresas, ya que disponen de mayor libertad y objetividad.

En cuanto a los consejeros extranjeros, los datos indican que su presencia en los CA influye en un número menor de valores éticos. Así, al igual que la diversidad de género, afecta especialmente a los valores de la categoría principios corporativos, como la “transparencia”, “rendición de cuentas” y “diversidad de género". Así pues, este resultado es consistente con el trabajo de Oxelheim et al. (2013), en el que se evidencia la influencia de los consejeros extranjeros en la toma de decisiones e iniciativas voluntarias del CA.

\section{CONCLUSIONES}

Los resultados obtenidos indican que principalmente las empresas latinoamericanas elaboran los códigos de conducta con valores éticos que tratan de mejorar las relaciones con sus stakeholders, abogando por la promoción de valores como la transparencia, integridad/confianza y responsabilidad. De este modo parece que, en línea O’Dwyer y Madden (2006), las empresas latinoamericanas muestran interés hacia el fomento de valores asociados con el compromiso social de la organización. No obstante, también prevalece el establecimiento de valores que tratan de proteger a la organización del empleado. Por tanto, la postura "defensiva" por la que inicialmente fueron creados los códigos de ética (Stevens, 1996) aún forma parte de los objetivos de los códigos éticos latinoamericanos. En este sentido, los resultados señalan la elevada promoción de conductas que reduzcan la malversación de fondos públicos y el uso de información interna para beneficio propio.

Por otro lado, cabe destacar que las empresas latinoamericanas son conscientes de la importancia de garantizar el cumplimento de los valores establecidos por la empresa, de acuerdo con autores como Kaptein (2004). A este respecto, vale señalar que tratan de "estimular" el cumplimiento de los valores establecidos por la organización a través de la imposición de sanciones 0 , dicho en otras palabras, tratan de controlar las buenas prácticas de la organización con medidas coactivas.

Por otro lado, los resultados indican que la composición del CA es un factor clave en el desarrollo del contenido de los códigos de ética. En particular, se observa que la mujer influye en los principios corporativos que fomentan la eficiencia, rendición de cuentas, lealtad y la diversidad de género en la organización. Además, se ha detectado que la presencia de mujer en el CA contribuye a la promoción de valores que velen por el cuidado de las relaciones con los clientes y, sobre todo, a inculcar la importancia de la calidad de los servicios que la empresa ofrece.

Del mismo modo, se cumplen las expectativas de la presencia de consejeros independientes, que son unos de los miembros del CA que fomentan la mejor elaboración de los códigos de ética. Con base en la teoría de la legitimidad, es posible que tanto la mujer como los consejeros independientes sean más conscientes de que para ser percibidos como "buenos" es tremendamente necesaria la tenencia de códigos de ética adecuados, que realmente sirvan a la consecución de un verdadero comportamiento responsable. Además, la presencia de consejeros independientes afecta en la implementación de las sanciones e incentivos en su conjunto. Asimismo, su presencia en el CA está asociada a valores como derechos humanos y justicia/ imparcialidad política en los códigos de conducta.

En el caso de los consejeros extranjeros, los datos indican que la presencia de consejeros de otro país reduce la atención en la mejora de los códigos de ética. En concreto, afectan en lo relativo a la rendición de cuentas y diversidad de género. En línea con la teoría de los stakeholders, es posible que este resultado se deba a que los consejeros extranjeros den mayor prioridad a otros temas de gestión de la organización, que a su parecer tengan una rentabilidad más a corto plazo con sus principales stakeholders. 
De acuerdo con los resultados obtenidos, este trabajo presenta varias contribuciones a nivel académico y práctico. A nivel académico, contribuye a la escasez de trabajos relacionados con los valores que son considerados por los códigos de ética de las empresas latinoamericanas, así como al poco explorado conocimiento sobre cuáles son los valores más promocionados en las empresas atendiendo a la diversidad de la composición del CA. Del mismo modo, permite avanzar en la literatura existente sobre la importancia del CA en la ética de la organización y, en concreto, en el contexto latinoamericano.

A nivel práctico, este trabajo puede ser considerado como un indicativo para las empresas latinoamericanas a la hora de conocer cómo desde la composición del CA pueden mejorar la implantación de sus códigos de ética. En este contexto, las empresas latinoamericanas deberían promover políticas que fomenten la sensibilización de los miembros del CA a asumir compromisos sociales y garantizar el cumplimiento de los objetivos corporativos, entre estas, sería conveniente reforzar la formación y el reciclaje del CA en materia de conducta ética. Además, el CA debería aprobar medidas de selección que favorezcan la diversidad de género e independencia dentro de su composición, dado el impacto positivo que tienen este tipo de consejeros en la inclusión de códigos de ética adecuados para garantizar las buenas prácticas de la organización. Por tanto, con la aplicación de estas nuevas medidas de selección, que están en línea con las recomendaciones sobre composición del CA existentes en el ámbito europeo, se podría lograr una proporcionalidad adecuada en la estructura del CA en Latinoamérica.

Como futuros trabajos de investigación, sería muy interesante avanzar en el grado de eficacia logrado por los códigos de ética y el CA en la mejora de la ética en las empresas latinoamericanas. En este sentido, sería muy fructífero conocer si el comportamiento corrupto de las empresas latinoamericanas está condicionado por la existencia o no de normas éticas. Además, con ello se podría esclarecer si aplicar un código de ética en la empresa se materializa en buenas prácticas y no al contrario, es decir, que las prácticas que llevan a cabo las empresas condicionan los contenidos de su código ético. Asimismo, sería interesante examinar la relación entre ética y economía para evaluar si las empresas que llevan a cabo buenas prácticas, con el propósito de evitar conflictos de intereses privados con los que persigue la empresa, son las que obtienen una mayor rentabilidad para accionistas e inversores. Por último, dado que en este estudio se identifican los contenidos más importantes a considerar en el código de conducta desde la perspectiva de la organización, quedaría pendiente explorar los distintos stakeholders externos clave de la organización, como clientes y proveedores.

\section{REFERENCIAS}

Abbott, W. F., \& Monsen, R. J. (1979). On the measurement of corporate social responsibility: Self-reported disclosures as a method of measuring corporate social involvement. The Academy of Management Journal, 22(3), 501-515.

Abrahamson, M. (1983). Social research methods. New Jersey, USA: Prentice Hall.

Adelstein, J., \& Clegg, S. (2016). Code of ethics: A stratified vehicle for compliance. Journal of Business Ethics, 168(1), 53-661-14. doi:10.1007/s10551-015-2581-9

Archel, P. (2003). La divulgación de la información social y medioambiental de la gran empresa española en el período 1994-1998: Situación actual y perspectivas. Revista Española de Financiación y Contabilidad, 32(117), 571-601. doi:10.1080/0210241 2.2003.10779496

Arruda, C. (1997). Business ethics in Latin America. Journal of Business Ethics, 16(14), 1597-1603. doi:10.1023/A:1005823317019

Arruda, C. (2009). Ethics and corporate social responsibility in Latin American small and medium sized enterprises: Challenging development. African Journal of Business Ethics, 4(2), 37-47. doi:10.15249/4-2-65

Ayuso, S. (2013). Códigos éticos de las empresas públicas españolas: Principios, conductas e implantación (Documento de trabajo no16). Recuperado de http://mango.esci.es/assets/Uploads/Documentos de-trabajo/Documento-de-trabajo 16Cdigo s-ticos-empresas-pblicas. pdf

Barrientos, S. W. (2005). Impact assessment and labour: Developing a learning approach. Journal of International Development, 17(2), 259270. doi:10.1002/jid.1213

Bear, S., Rahman, N., \& Post, C. (2010). The impact of board diversity and gender composition on corporate social responsibility and firm reputation. Journal of Business Ethics, 97(2), 207-221.doi:10.1007/ s10551-010-0505-2

Bedicks, H. B., \& Arruda, C. (2005). Business ethics and corporate governance in Latin America. Business and Society, 44(2), 218-228. doi:10.1177/0007650305275301

Berrone, P., Surroca, J., \& Tribó, J. A. (2007). Corporate ethical identity as a determinant of firm performance: $A$ test of the mediating role of stakeholder satisfaction. Journal of Business Ethics, 76(1), 35-53. doi:10.1007/s10551-006-9276-1

Bondy, K., Matten, D., \& Moon, J. (2004). The adoption of voluntary codes of conduct in MNCs: A three-country comparative study. Business and Society Review, 109(4), 449-477. doi:10.1111/j.00453609.2004.00205.x

Briano, G. C., \& Saavedra, M. L. (2015). La composición del consejo de administración y la estructura accionaria como factores explicativos de la transparencia en el gobierno corporativo en Latinoamérica: Evidencia en empresas cotizadas de Argentina, Brasil, Chile y México. Estudios Gerenciales, 31(136), 275-286.

Calderón, M. (2011). CSR in Latin America and South East Asia analysis of the corporate communication of top local companies. International Research Journal of Finance and Economics, 73, 56-74. 
Cárdenas, M. C., Eagly, A., \& Salgado, E., Goode, W., Heller, L. I., Jauregui, K., ... Tunqui, R. C. (2014). Latin American female business executives: An interesting surprise. Gender in Management: An International Journal, 29(1), 2-24. doi:10.1108/GM-06-2013-0067

Comisión Nacional del Mercado de Valores. (2015). Código de buen gobierno de las sociedades cotizadas. Comisión Nacional del Mercado de Valores. Recuperado de http://www.cnmv.es/DocPortal/ Publicaciones/CodigoGo v/Codigo_buen_gobierno.pdf

Conyon, M. J., \& Mallin, C. (1997). Women in the boardroom: Evidence from large UK companies. Corporate Governance: An International Review, 5(3), 112-117. doi:10.1111/1467-8683.00051

Coonjohn, J. J., \& Lodin, A. (2011). Developing a strategic implementation plan for anticorruption. Recuperado de http://www.jjcoonjohn.com/ pdf/Strategic_Implementation_Plan.pdf

Criado-Jiménez, I., Fernández-Chulián, M., Husillos-Carqués, F. J., \& Larrinaga-González, C. (2008). Compliance with mandatory environmental reporting in financial statements: The case of Spain (2001-2003). Journal of Business Ethics, 79(3), 245-262. doi:10.1007/ S10551-007-9375-7

Cuadrado-Ballesteros, B., García-Rubio, R., \& Martínez-Ferrero, J. (2015). Efecto de la composición del consejo de administración en las prácticas de responsabilidad social corporativa. Revista de Contabilidad, 18(1), 20-31. doi:10.1016/j.rcsar.2014.02.003

Elias, J. (2003). International labour standards, codes of conduct and gender issues: A review of recent debates and controversies. Non-State Actors and International Law, 3(2-3), 283-301. doi:10.1163/157180703322765102

Erwin, P. M. (2010). Corporate codes of conduct: The effects of code content and quality on ethical performance. Journal of Business Ethics, 99(4), 535-548, 2010. doi:10.1007/s10551-010-0667-y

Francoeur, C., Labelle, R., \& Sinclair-Desgagné, B. (2008). Gender diversity in corporate governance and top management. Journal of Business Ethics, 81(1), 83-95. doi:10.1007/s10551-007-9482-5

Frías-Aceituno, J. V., Rodríguez-Ariza, L., \& García-Sánchez, I. M. (2013). The role of the board in the dissemination of integrated corporate social reporting. Corporate Social Responsibility and Environmental Management, 20(4), 219-233. doi:10.1002/csr.1294

Gálvez-Rodríguez, M., Caba-Pérez, C., \& López-Godoy, M. (2015). Enhancing online information of African nongovernmental organizations through self-regulation: National experiences versus international initiatives. Information Development. doi:10.1177/0266666914568392.

García-Sánchez, I. M., Rodríguez-Domínguez, L., \& Frías-Aceituno, J. V. (2015). Board of directors and ethics codes in different corporate governance systems. Journal of Business Ethics, 131(3), 681-698, 2014. doi:10.1007/s10551-014-2300-y

Gilley, K. M., Robertson, C. J., \& Mazur, T. C. (2010). The bottom-line benefits of ethics code commitment. Business Horizons, 53(1), 31-37. doi:10.1016/j.bushor.2009.08.005

González, E. (2007). La teoría de los stakeholders: Un puente para el desarrollo práctico de la ética empresarial y de la responsabilidad social corporativa. Veritas, 2(17), 205-224.

Gorbaneff, Y., Uribe, E. M., \& Hoyos, G. (2012). Los códigos de conducta de las empresas petroleras en Colombia: Una caracterización. Revista de Economía Institucional, 14(27), 193-205.
Graneheim, U. H., \& Lundman, B. (2004). Qualitative content analysis in nursing research: Concepts, procedures and measures to achieve trustworthiness. Nurse Education Today, 24(2), 105-112. doi:10.1016/j.nedt.2003.10.001

Guthrie, J., \& Parker, L. D. (1990). Corporate social disclosure practice: A comparative international analysis. Advances in Public Interest Accounting, (3), 159-175.

Hackston, D., \& Milne, M. J. (1996). Some determinants of social and environmental disclosures in New Zealand companies. Accounting, Auditing and Accountability Journal, 9(1), 77-108. doi:10.1108/09513579610109987

Kaptein, M. (2004). Business codes of multinational firms: What do they say? Journal of Business Ethics, 50(1), 13-31. doi:10.1023/ B:BUSI.0000021051.53460.da

Kaptein, M., \& Schwartz, M. S. (2008). The effectiveness of business codes: A critical examination of existing studies and the development of an integrated research model. Journal of Business Ethics, $77(2)$, 111-127. doi:10.1007/s10551-006-9305-0

Kaptein, M., \& Wempe, J. (2002). The balanced company: A theory of corporate integrity. Oxford, UK: Oxford University Press.

Krippendorff, K. (1980). Content analysis: An introduction to its methodology. California, USA: Sage.

Li, J., Pike, R., \& Haniffa, R. (2008). Intellectual capital disclosure and corporate governance structure in UK firms. Accounting and Business Research, 38(2), 137-159. doi:10.1080/00014788.2008.9663326

Long, B. S., \& Driscoll, C. (2008). Codes of ethics and the pursuit of organizational legitimacy: Theoretical and empirical contributions. Journal of Business Ethics, 77(2), 173-189. doi:10.1007/ s10551-006-9307-y

Lückerath-Rovers, M., \& Bos, A. De. (2011). Code of conduct for nonexecutive and supervisory directors. Journal of Business Ethics, 100(3), 465-481. doi:10.1007/s10551-010-0691-y

Luo, Y. (2005). How does globalization affect corporate governance and accountability? A perspective from MNEs. Journal of International Management, 11(1), 19-41. doi:10.1016/j.intman.2004.11.003

Mateos de Cabo, R., Gimeno, R., \& Nieto, M. J. (2012). Gender diversity on European banks' boards of directors. Journal of Business Ethics, 109(2), 145-162. doi:10.1007/s10551-011-1112-6

Méndez, J. L. S., \& Gómez, E. A. (2013). Las funciones de los consejeros independientes de las sociedades en México: recomendaciones para el cambio a partir de las asimetrías existentes entre la práctica y la ley. Boletín Mexicano de Derecho Comparado, 46(136), 287-316. doi: 10.1016/Soo41-8633(13)71128-1

Nachtsheim, C. J., Neter, J., Kutner, M. H., \& Wasserman, W. (2004). Applied linear regression models. Irwin, USA: McGraw-Hill.

Nekhili, M., \& Gatfaoui, H. (2013). Are demographic attributes and firm characteristics drivers of gender diversity? Investigating women's positions on French boards of directors. Journal of Business Ethics, 118(2), 227-249. doi:10.1007/s10551-012-1576-z

O’Dwyer, B., \& Madden, G. (2006). Ethical codes of conduct in Irish companies: A survey of code content and enforcement procedures. Journal of Business Ethics, 63(3), 217-236. doi:10.1007/s10551-0053967-x

Office of Transport Safety Investigations. (2010). Fraud and corruption prevention strategy. Office of Transport Safety Investigations. Recuperado de www.otsi.nsw.gov.au/access-to-info/OTSI-FraudStrategy.pdf 
Oxelheim, L., Gregorič, A., Randøy, T., \& Thomsen, S. (2013). On the internationalization of corporate boards: The case of Nordic firms. Journal of International Business Studies, 44(3), 173-194. doi:10.1057/jibs.2013.3

Pfeil, U., Arjan, R., \& Zaphiris, P. (2009). Age differences in online social networking: A study of user profiles and the social capital divide among teenagers and older users in MySpace. Computers in Human Behavior, 25(3), 643-654. doi:10.1016/j.chb.2008.08.015

Prado-Lorenzo, J. M., \& García-Sánchez, I. M. (2010). The role of the board of directors in disseminating relevant information on greenhouse gases. Journal of Business Ethics, 97(3), 391-424. doi:10.1007/s10551-010-0515-0

Prieto, M., \& Bendell, J. (2002). If you want to help us then start listening to us! From factories and plantations women speak out about corporate responsibility. Bristol, UK: New Academy of Business.

Prieto, M., Hadjipateras, A., \& Turner, J. (2002). The potential of codes as part of women's organisations' strategies for promoting the rights of women workers: A CentralAmerica perspective. In R. Jenkins, R. Pearson, \& G. Seyfang (Eds.), Corporate responsibility and labour rights: Codes of conduct in the global economy. London, UK: Earthscan.

Prieto-Carrón, M. (2008). Women workers, industrialization, global supply chains and corporate codes of conduct. Journal of Business Ethics, 83, 5-17. doi:10.1007/s10551-007-9650-7

Roblyer, M. D., McDaniel, M., Webb, M., Herman, J., \& Witty, J. V. (2010). Findings on Facebook in higher education: A comparison of college faculty and student uses and perceptions of social networking sites. The Internet and Higher Education, 13(3), 134-140. doi:10.1016/j. iheduc.2010.03.002

Rodríguez-Ariza, A., Frías-Aceituno, J. L., \& García-Rubio, R. (2014). El consejo deadministración y las memorias de sostenibilidad. Revista de Contabilidad, 17(1), 5-16. doi:10.1016/j.rcsar.2013.02.002

Rodríguez-Domínguez, L., Gallego-Álvarez, I., \& García-Sánchez, I. M. (2009). Corporate governance and codes of ethics. Journal of Business Ethic, 90, 187-202. doi:10.1007/s10551-009-0035-y

Schwartz, M. S. (2002). A code of ethics for corporate code of ethics. Journal of Business Ethics, 41(1-2), 27-43.

Schwartz, M. S., Dunfee, T. W., \& Kline, M. J. (2005). Tone at the top: An ethics code for directors? Journal of Business Ethics, 58, 79-100. doi:10.1007/s10551-005-1390-y
Sharbatoghlie, A., Mosleh, M., \& Shokatian, T. (2013). Exploring trends in the codes of ethics of the fortune 100 and global 100 corporations. The Journal of Management Development, 32(7), 675689. doi:10.1108/JMD-04-2011-0044

Sheskin, D. J. (2011). Handbook of parametric and nonparametric statistical procedures (5th ed.). Boca Raton, USA: Chapman \& Hall/ CRC.

Singh, J., Carasco, E., Svensson, G., Wood, G., \& Callaghan, M. (2005). A comparative study of the contents of corporate codes of ethics in Australia, Canada and Sweden. Journal of World Business, 40(1), 91109. doi:10.1016/j.jwb.2004.10.007

Soltani, B., \& Maupetit, C. (2015). Importance of core values of ethics, integrity and accountability in the European corporate governance codes. Journal of Management and Governance, 19(2), 259-284. doi:10.1007/S10997-013-9259-4

Stevens, B. (1996). Using the competing values framework to assess corporate ethical codes. Journal of Business Communication, 33(1), 71-84. doi:10.1177/002194369603300107

Valentine, S., \& Fleischman, G. (2008). Ethics programs, perceived corporate social responsibility and job satisfaction. Journal of Business Ethics, 77(2), 159-172. doi:10.1007/s10551-006-9306-z

Webb, E. (2004). An examination of socially responsible firms' board structure. Journal of Management and Governance, 8(3), 255-277. doi:10.1007/s10997-004-1107-0

Weiss, A. (1991). Seven reasons to examine workplace ethics. $H R$ Magazine, 36(3). Recuperado de http://www.hrmagazine.co.uk/

Wood, G. (2000). A cross cultural comparison of the contents of codes of ethics: USA, Canada and Australia. Journal of Business Ethics, 25(4), 287-298. doi:10.1023/A:1006034209956

Wotruba, T. R., Chonko, L. B, \& Loe, T. W. (2001). The impact of ethics code familiarity on manager behavior. Journal of Business Ethics, 33(1), 59-69. doi:10.1023/A:1011925009588

Zhang, J. Q., Zhu, H., \& Ding, H. B. (2013). Board composition and corporate social responsibility: An empirical investigation in the post Sarbanes-Oxley era. Journal of Business Ethics, 114(3), 381-392. doi:10.1007/s10551-012-1352-0 